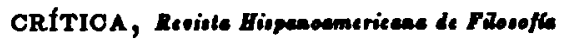

Vol. XX, No. 80 (egosto 1088): 8-21

\title{
EMOCIONES REACTIVAS
}

OLBETH HANSBERG

Instituto de Investigaciones Filosofficas, UNAM

En su artículo "Freedom and Resentment" Strawson nos invita a pensar en "los múltiples tipos de relaciones que podemos tener con otras personas - cuando compartimos un interés mutuo; como miembros de la misma familia; como colegas, amigos, amantes; como partes ocasionales de un sinnúmero de transacciones y encuentros. Y, frente a cada una de estas conexiones y otras posibles, debemos pensar después en la importancia que le conferimos a las actitudes e intenciones que tienen hacia nosotros aquellas personas que se relacionan con nosotros de esa manera, y en los tipos de actitudes y sentimientos reactivos a los que nosotros mismos somos propensos".

En lo que sigue me propongo examinar este tipo de emociones que, como respuesta a las actitudes, intenciones y acciones de otras personas, son emociones esencialmente sociales. Dado el hecho de la sociedad humana y de nuestro status como seres sociales, nos vemos involucrados y establecemos múltiples relaciones con otros seres humanos. En esta interacción adquieren una im-

1 P. F. Strawson, Freedom and Resentment and Olker Esonys, Londres, Methuen, 1974, p. 6. La discusión de Strawson se da en el marco de una problemática distinta: la del determinismo y la posibilidad de la responsabilidad. Yo, en cambio, me referiré en este trabajo exclusivamente a su caracterización de los sentimientos y actitudes reactivos. 
portancia central las actitudes y sentimientos que tienen hacia nosotros los demás - su buena o mala voluntad, su indiferencia - y las actitudes y sentimientos con los que nosotros reaccionamos a las acciones y actitudes de otras personas. Así, reaccionamos con resentimiento, admiración o disgusto - censuramos o alabamos una acción; nos indigna o aprobamos el trato que tienen unos con otros los seres humanos; y nos arrepentimos, nos avergonzamos o nos autocomplacemos por la manera en que nosotros mismos actuamos frente a ellos.

En nuestra vida en comunidad participamos y establecemos relaciones interpersonales de tipos muy diversos - de las más íntimas a las más casuales-, pero en general exigimos que éstas se den en un clima de buena voluntad y respeto mutuo, aunque las formas específicas en las que se manifiesten dependan del tipo de relación y de las circunstancias en las que se establezcan. Las actitudes y sentimientos reactivos son una respuesta a la buena o mala voluntad o a la indiferencia que manifiestan las personas en sus actitudes y conducta hacia las personas con las que se relacionan. ${ }^{2}$

Strawson distingue varios tipos de actitudes y sentimientos reactivos: los personales, los impersonales o morales y los autorreactivos. Más adelante me ocuparé de estas distinciones. Por ahora sólo me referiré a las actitudes y sentimientos personales que tiene una persona frente a las actitudes que tienen los demás hacia ella o hacia alguien con quien se identifica. Algunos ejemplos son: resentimiento, disgusto, agradecimiento, y actitudes de reproche, alabanza o censura...

Afirma que nuestra propensión a las actitudes y sentimientos reactivos personales "es un hecho natural, tejido en la trama de nuestras vidas, algo dado con el

2 op. cit., p. 6. 
hecho de la sociedad humana como la conocemos". Son actitudes y sentimientos que tienen raíces comunes en nuestra naturaleza humana y en nuestra pertenencia a comunidades humanas. Como totalidad, estos sentimientos y actitudes no requieren de una "justificación racional", esto es, no podemos explicar el que las tengamos, pero sí podemos reflexionar y entender cuáles son sus límites y por qué estos límites tienden a caer donde caen, cuáles circunstancias y condiciones las propician o inhiben. Podemos criticarlas en casos particulares, decir cuándo son apropiadas o razonables y en qué medida nos sirven para entender y explicar conductas individuales y prácticas sociales. ${ }^{3}$

Para examinar con mayor detalle los distintos elementos y condiciones, es conveniente que nos detengamos en unos ejemplos:

Dos personas A y B tiene cierta relación personal, digamos que son amigos. Esto implica al menos que cada uno tiene alguna idea de la personalidad y del temperamento del otro, y ciertas exigencias y expectativas acerca de su conducta y actitudes con él en diversos tipos de circunstancias. Ahora bien, en una ocasión dada A hace una acción particular a que daña u ofende a $B$. $B$. reconoce que la acción de $A$ lo ha dañado, o la ve como una acción ofensiva para él, y cree que es, o la ve como, la manifestación de una actitud de mala voluntad de parte de $A$ hacia él. $B$ reacciona con resentimiento hacia A y censura su acción. ${ }^{4}$

Supongamos ahora que $B$ expresa de alguna manera

3 P. F. Strrawson, "Reply to Bennett", en: Van Straaten (ed.), Philosophical Subjects, Oxford, 1980, p. 265.

- Doy versiones alternativas porque quiero distinguir entre 'creer' $y$ 'ver como'. No quiero comprometerme con la tesis de que siempre es una creencia especifica la que determina una emoción: cree que a le hace daño, cree que es ofensiva. Pienso que a veces puede ser más relevante para una emoción el 'ver a c como ofensiva'. Ver Olbeth 
su resentimiento. $A$ reconoce que su acción causó resentimiento en $B$, siente remordimiento, lamenta su acción y hace a su vez algo que $B$ pueda ver como una acción reparadora...

O, para mencionar el caso de una emoción positiva, $A$ hace algo que beneficia a $B$. B reconoce que la acción de A lo beneficia y que al hacerla, A manifiesta un grado de buena voluntad que supera sus expectativas. $B$ reacciona con agradecimiento hacia $\mathbf{A}$ y alaba su acción...

Estos ejemplos sólo muestran posibles secuencias en las que emociones y actitudes nos proporcionan un marco interpretativo que nos permite comprender y explicar la conducta de un individuo, no como un suceso aislado, sino dentro del sistema de relaciones humanas.

Podemos distinguir aquí varios elementos relacionados entre sí: el sistema de relaciones humanas, las exigencias y expectativas en las que se basa, su manifestación o no en actitudes y conductas y nuestra propensión a responder con sentimientos y actitudes reactivas. $^{\mathrm{s}}$

Empecemos por el núcleo constituido por la situación particular en la que $A$ hace algo que produce resentimiento en $\mathrm{B}$. Si $\mathrm{B}$ siente resentimiento por algo que hizo $A$, debemos suponer que $B$ piensa:

1) Que la acción de $A$ es intencional, esto es, que $A$ es responsable de su acción.

2) Que esa acción le ha producido algún daño, o que

Hansberg, “Emociones y creencias”, Revista Latinoamericana de Filosofia, 1988.

- Strawson menciona siempre juntos los sentimientos y las actitudes reactivas. No pretende proporcionar una definición de cada una, sino solo dar ejemplos. Intuitivamente, resentimiento, gratitud, indignación, etc., son centimientos - aprobar, censurar, culpar, valorar, etc., son actitudes. Pero los dos van juntos: cuando siento resentimiento, culpo a alguien y cenauro su acción. Habría sin embargo que precisar la relación entre ellos. 
es ofensiva, insultante o de alguna manera nociva para él.

3) Que esa acción nociva para $\mathrm{B}$ es, además, una manifestación de mala voluntad de parte de $A$, esto es, que al hacer esa acción $A$ ha querido ofender, insultar o dañar a B.

La distinción entre 2) y 3) es importante puesto que alguien puede hacer algo que daña $u$ ofende sin tener la intención de ofender o sin mostrar ninguna hostilidad o mala voluntad. Pero es central en una ocasión de resentimiento el que el sujeto resentido reconozca en la conducta del otro una actitud negativa hacia él.

1), 2) y 3) son condiciones que se dan cuando hay resentimiento; obviamente no son condiciones suficientes para que alguien sienta resentimiento. El que alguien sienta o no resentimiento en una ocasión dada "es en parte asunto de su temperamento individual, estilo personal, estado de ánimo del momento y quizá accidente fisiológico".6 No hay reglas que digan cuándo alguien debe sentir resentimiento, sino sólo condiciones en las que es posible que se dé el resentimiento y ciertas circunstancias en las que característicamente se da.

Ahora bien, parece que con respecto a las tres condiciones anteriores, lo importante es cómo ve las cosas el sujeto resentido, esto es, si ve al agente como plenamente responsable de su acción, si ve la acción como ofensiva y como una manifestación de mala voluntad del agente hacia él. De tal manera que si el sujeto se enterara de que las cosas no son en realidad como él piensa que son, esto constituiría una razón para suspender su resentimiento.

En relación con las tres condiciones anteriores, pode-

- J. Bennett, "Accountability", en: Van Straaten (ed.), op. cil., p. 23. 
mos mencionar varias maneras en que un sentimiento reactivo personal puede ser inapropiado o irracional. Pienso que cuando una persona se da cuenta de que su reacción es inapropiada, a saber, cuando tiene nuevos elementos que cambian su apreciación de la situación $y$, sin embargo, esto no modifica o hace desaparecer la emoción, entonces diremos que estamos frente a un caso de emoción irracional.

Strawson menciona varias consideraciones especiales en las que es inapropiado tener un sentimiento reactivo: ${ }^{7}$

1. Cuando tenemos sentimientos y actitudes reactivas ordinarias frente al agente, en general así como en un momento particular, es decir, cuando lo vemos como plenamente responsable, y, sin embargo, en cierta ocasión específica, no es responsable de una ofensa o daño particular. Se trata aquí de situaciones a las que son aplicables frases como "no se dio cuenta", "no sabía", "fue un accidente", etcétera.

2. Cuando suspendemos en general o momentáneamente nuestras actitudes reactivas frente al agente:

a) porque se dan circunstancias especiales en que vemos al agente bajo una luz distinta que excluye estas emociones. Por ejemplo, cuando adoptamos el punto de vista objetivo, esto es, cuando nos desligamos del compromiso de una relación participativa y vemos al agente como un 'caso';'

b) cuando las circunstancias son normales pero el agente es anormal, o sea, cuando no está capacitado para tener relaciones interpersonales porque no es responsable de sus acciones. Así, es inapropiado tener sentimientos reactivos frente a un loco, un bebé, un re-

7 Strawson, Freedom and Resentment and Other Essays, pp. 7-10.

op. cit., sección IV. 
trasado mental... o, en ciertos momentos, frente a un borracho, una persona drogada, etcétera.

Los casos que menciona Strawson son aquellos en los que $o$ no tenemos sentimientos o actitudes reactivas (2a), o es inapropiado tenerlos ( 1 y 2 b). La impropiedad de estos últimos se refiere a la primera condición: la que afirma que es apropiado tener sentimientos reactivos frente a un agente responsable y cuando su acción fue intencional.

Pero me parece que hay otros casos en los que los sentimientos y actitudes reactivas pueden verse como inapropiados, que tienen que ver con las otras dos condiciones. Estos casos son elusivos y difíciles de expresar, pues tienen que ver con las relaciones que existen entre cómo es la realidad y las interpretaciones que de ella hace el sujeto. Daré algunos ejemplos:

En relación con 2) podemos distinguir varios casos:

a) cuando $B$ reacciona con resentimiento porque cree falsamente que A hizo algo que no hizo;

b) cuando $B$ cree falsamente que lo que hizo $A$ lo daña cuando en realidad la acción de $A$ no resulta en un daño objetivo;

c) cuando $B$ reacciona frente a una ofensa imaginaria. Éste es un caso más pantanoso porque no es fácil de decidir. Calificar la ofensa de "imaginaria" significa decir que $B$ ve una ofensa donde nadie más la ve -o quizá que la acción de $A$ no tiene ninguna característica que justifique el que $B$ la interprete como ofensiva. Pero lo que alguien pueda ver como ofensivo o insultante dependerá en gran medida de su personalidad, del momento y de su apreciación de la situación.

Aquí se abren varias posibilidades para calificar de inapropiado el ver como ofensiva una conducta. Digamos que $B$ tiene una idea tan exagerada de su propia importancia que considera como una ofensa cualquier 
conducta que implique la más ligera desviación de su persona como foco de atención. O supongamos, alternativamente, que tiene tal inseguridad que casi cualquier cosa puede afectar su autoestima. Por otro lado, podemos pensar también que está tan irritado o susceptible que ve ofensas donde no las hay, o que su apreciación de la situación es de alguna otra manera equivocada.

En relación con la condición 3) hay también casos en que la reacción puede ser inapropiada. Una posibilidad es que le atribuya al agente actitudes que no tiene. Así, en el caso del resentimiento, veo la acción de $A$ como la manifestación de una actitud de mala voluntad hacia $B$ que A no tiene; o veo como indiferencia lo que en realidad es una distracción momentánea, o como hostilidad lo que es sólo impaciencia.

Aquí, como en el caso anterior, la atribución de mala voluntad a una persona dependerá, en parte, también del conocimiento que tiene el sujeto de ella, de sus expectativas con respecto a ella y de la situación específica. Esto puede dar lugar a atribuciones erróneas de actitudes debido, por ejemplo, a un conocimiento defectuoso de la persona o a una apreciación equivocada de la situación.

En general, la persona que ve como ofensiva una ac. ción, atribuirá mala voluntad al agente que considera responsable de esa acción. Esto es así, en parte, porque nosotros, cuando queremos ofender o hacer daño, comúnmente haremos algo que la otra persona pueda reconocer como una ofensa o un daño.

Ahora bien, creo con Strawson y en contra de Bennett $^{9}$ que los sentimientos reactivos personales requieren de relaciones personales antecedentes a la ocasión actual, y que no basta con el contacto que hay cuando se

- Bennett, op. cit., pp. 43-5. 
da la reacción. Yo diría que si alguien tiene una actitud de buena o mala voluntad hacia mi, esto implica que hay una relación antecedente, por más lejana que ésta sea. El ejemplo de Bennett de un benefactor desconocido frente al cual siento agradecimiento, no funciona a menos que yo piense que el beneficio que me otorgó muestra una actitud de buena voluntad hacia mí. Mi agradecimiento está ligado no sólo al beneficio que obtuve, sino también y sobre todo a la persona que al beneficiarme muestra su buena voluntad hacia mí. Si me entero, por ejemplo, de que el beneficio no está dirigido a mí, sino que me tocó sólo como resultado de un sorteo, y que el benefactor no tiene ninguna idea acerca de mi persona, lo más probable es que no sienta agradecimiento sino sólo alegría por mi buena suerte, y que alabe la generosidad de mi benefactor.

Sin embargo, como se desprende del ejemplo anterior, una relación previa no implica necesariamente que las personas relacionadas hayan tenido un trato personal. Para que alguien pueda responder con un sentimiento o actitud reactiva personal como gratitud o resentimiento, basta con que tenga cierto conocimiento, aunque sea indirecto, de la persona, de tal manera que pueda atribuirle actitudes positivas o negativas dirigidas hacia él o hacia alguien con el cual se identifica. Y, por otro lado, para poder tener actitudes positivas o negativas hacia una persona específica debo suponer que existe alguna relación y, por tanto, algún conocimiento de la persona frente a la cual tengo la actitud.

Las emociones reactivas personales son emociones que sentimos primordialmente frente a personas. El resentimiento, por ejemplo, requiere que haya alguien a quien culpar por una acción que censuramos: estoy resentido con Juan porque no votó por mí; resiento el que Juan me criticara en público. Aun en los casos en que no sé 
exactamente a quién culpar, estoy implicando la existencia de algún culpable: resiento el que no me eligieran, resiento.el que nadie me escuche. En todos los casos la mención, aunque sea implícita, de alguna persona o personas responsables de lo que considero ofensivo, parece ineludible.

No resiento, en cambio, el hecho de que mi coche no arrancara esta mañana, ni tampoco siento resentimiento con el niño que llora todo el tiempo y no me deja trabajar, ni con el perro que me mordió. Tampoco parece claro que podamos resentirnos con una persona extraña con la cual no tenemos ningún tipo de relación y que, por ejemplo, nos dice una grosería en la calle. En todos estos casos el enojo sería quizá una emoción más adecuada. ${ }^{10}$

Ahora bien, tener la propensión a actitudes y sentimientos reactivos es tener exigencias y expectativas frente a otras personas con las cuales convivimos en una comunidad y con las cuales establecemos relaciones personales. Según Strawson, lo que en general hace posible esta convivencia y los distintos tipos de relaciones en las que estamos involucrados, es la exigencia que tenemos, los unos frente a los otros, de buena voluntad y de respeto a la integridad personal. Éste es un

\footnotetext{
10 Aunque en muchos casos se usan indistintamente, el enojo parece distinto al resentimiento en este sentido. El enojo no requiere que alguna persona tenga una actitud especffica hacia mí. Aparentemente basta, cuando me enojo con alguien, que haga algo que me afecte negativamente. Tampoco se requieren aqui relaciones previas: puedo enojarme con un perro, con el niño, el loco, o con un extraño que me insulta. También puedo enojarme por una situación que me afecta sin que importe que haya o no alguien a quien culpar. Y ni siquiera, como en el caso del resentimiento, es necesaria la referencia a mi, ya que puedo enojarme, por ejemplo, por un acto ofensivo dirigido a otra persona o a un animal. O cuando alguien daña una obra de arte o destruye una flor. Habría que distinguir más claramente entre enojo, resentimiento e indignación. Son emociones distintas a pesar de que en muchas situaciones son aplicables las tres y no se puede distinguir fácilmente entre ellas.
} 
requisito muy general que tendrá que manifestarse en formas muy diversas que dependerán, entre otras cosas, de las características de la comunidad o comunidades a las que pertenecemos, del tipo de relación interpersonal, de la historia particular de la relación, de las circunstancias particulares y de variaciones individuales de las personas.

Así, si A y B son amigos, tendrán una serie de expectativas y exigencias más o menos explícitas que provienen de cómo ve cada uno la relación en la que participan y del conocimiento más o menos claro que puedan tener el uno del otro. Esto incluirá ciertas ideas acerca de su personalidad y constitución emocional en general, que les permitirán vislumbrar, por ejemplo, qué tipo de cosas son las que provocan su resentimiento, o lo complacen, lo admiran o lo indignan. Todo esto forma un trasfondo que en un momento dado nos sirve para situar una determinada actitud en una red de otras actitudes y estados mentales. Constituye un marco explicativo que en determinada ocasión nos permite entender, por ejemplo, qué es lo que $B$ espera de su amigo, qué es lo que desea que haga o deje de hacer y qué es lo que, en un caso específico, puede ver como una manifestación de hostilidad o indiferencia.

En relación con lo anterior puedo hablar también de reacciones emotivas inapropiadas. Me refiero a aquellas que se basan en una apreciación errónea de la personalidad del otro, de la relación personal o de las expectativas y exigencias que es razonable tener con respecto a ella. Así, si creo que alguien es mi amigo cuando no lo es, puedo tener exigencias y expectativas que no corresponden a la relación que de hecho existe. Esto puede dar lugar a frustraciones de deseos y expectativas que pueden ser causa de resentimiento, y que no tendrían por qué surgir si tuviéramos una apreciación más ade- 
cuada del tipo de relación. O supongamos, por último, que mi apreciación de la relación es correcta, digamos que sí somos amigos, pero mis exigencias con respecto a la otra persona son tan desproporcionadas a la situación o mis expectativas tan irreales, que haga lo que haga el otro no podrá estar a la altura de ellas. ${ }^{11}$

Existen, entonces, múltiples razones por las cuales una emoción pueda considerarse como inapropiada. He mencionado aquí algunas que tienen que ver con las distintas maneras en que el sujeto que tiene el sentimiento reactivo puede equivocarse en su apreciación del agente, de la acción, de las actitudes que la causaron, del tipo de relación y de las exigencias y expectativas en las que se basa.

Hasta ahora he tratado de examinar las condiciones en las que pueden darse los sentimientos y actitudes reactivas personales y he mencionado algunos casos en los que pueden ser inapropiados. Para finalizar haré un breve comentario muy general acerca del papel que juegan las emociones de este tipo en la explicación de la conducta de un individuo.

Como hemos dicho anteriormente, las explicaciones que mencionan sentimientos y actitudes reactivas sitúan a la conducta de un individuo en un contexto social. El interés de la explicación se centra no en una acción aislada sino en la conducta como respuesta a las acciones, actitudes y emociones de otras personas cuyas conductas, a su vez, se ven como respuestas a otras acciones, actitudes y emociones nuestras y de los demás.

Por otro lado, reconocer la emoción que tiene una

11 No pretendo que éstas sean las únicas razones para calificar de inapropiado un sentimiento reactivo. Otra forma en que puede ser inapropiado es cuanda el sentimiento mismo es demasiado intenso en proporción a su causa o cuando dura demasiado. Esto es sumamente vago y habría que precisarlo mucho más, quizá mediante ejemplificaciones detalladas. 
persona en un momento dado puede guiar mi conducta - como cuando cambio la conversación al ver que está causando perplejidad en el otro. ${ }^{12}$ Tener una idea acerca del tipo de cosas que a alguien le agradan, le molestan, le disgustan, le indignan... nos permite entender por qué hace o deja de hacer ciertas cosas y nos ayuda a regular nuestra propia conducta con él de tal manera que podamos promover ciertas actitudes y tratar de inhibir otras.

Pero sobre todo la referencia a emociones nos permite unificar y, por tanto, entender y explicar todo un conjunto de acciones muy variadas y aparentemente inconexas que, sin embargo, responden a un esquema de comportamiento. Ejemplo de esto son las cosas muy diferentes que puede hacer alguien que se arrepiente de una acción y que pueden verse como expresiones de su culpabilidad y que pretenden reparar el daño causado.

Mencionar una emoción puede ser también una forma de unificar un gran número de deseos específicos al ver que tienen un origen común en una emoción determinada. Por ejemplo, los deseos que tiene una persona de hacer cosas distintas que expresen su amor o su odio por alguien. Una gran desilusión puede a veces explicar el cambio de rumbo de una vida y una vida entera puede verse como la expiación de un crimen.

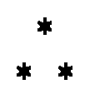

Para terminar me referiré brevemente a los otros dos tipos de emociones reactivas que menciona Strawson: las autorreactivas y las morales o impersonales.

Las primeras son las que tiene una persona ante sus propias acciones o actitudes frente a los demás, como

12 Strawson, “Reply to Bennett”, p. 264. 
por ejemplo el sentirse arrepentido, avergonzado, autocomplacido o culpable. Son emociones con las que una persona reacciona ante el reconocimiento de que su actitud o acción fue dañina, ofensiva o benéfica para otra u otras personas; o que fue una manifestación de hostilidad, indiferencia o buena voluntad hacia ella(s). Son emociones que nos permiten entender que alguien pueda verse a sí mismo como merecedor de un reproche, un castigo o una recompensa. Un sentimiento autorreactivo como el remordimiento nos permite, por ejemplo, explicar una acción o un conjunto de acciones como la reparación de una ofensa, como la aceptación de una culpa o como un autocastigo.

El otro tipo de actitudes y sentimientos reactivos es el que Strawson llama 'morales' y que describe como "los análogos simpáticos o vicarios o desinteresados o generalizados" de las actitudes reactivas personales. ${ }^{13}$ Se trata de sentimientos y actitudes reactivas como indignación, desaprobación o aprobación morales. Pero veamos con más detalle cuáles son sus rasgos característicos:

A diferencia de las actitudes y sentimientos reactivos personales, no está en estos casos involucrado "el interés o la dignidad" de la persona que tiene la reacción, esto es, se trata de la reacción que tiene una persona frente a las acciones y actitudes que tienen los demás hacia otros seres humanos. Son análogos "vicarios" de las actitudes reactivas personales: así, la indignación, por ejemplo, es resentimiento en nombre de, o por, otro.

Estas actitudes y sentimientos impersonales "se apoyan en, y reflejan", la misma exigencia de que los demás manifiesten en sus relaciones "un grado razonable de buena voluntad o aprecio". Pero ahora la exigencia ten-

13 Strawson, "Freedom and Resentment", p. 14. 
drá una "forma generalizada", a saber, la buena voluntad no estará dirigida especialmente a nosotros sino a todos los seres humanos en general. ${ }^{14}$ Desaprobación moral e indignación son respuestas a las actitudes y conducta de alguien que, en su trato con otros seres humanos, no satisface esta demanda o actúa en contra de ella.

Pero detengámonos un momento en el primero de los rasgos que he mencionado: las actitudes y sentimientos morales son análogos vicarios de las actitudes reactivas personales - la indignación es resentimiento en nombre de, o por, otra persona. Pero ¿qué es lo que quiere decir esto? Una manera de verlo es decir que indignación es algo que tengo propensión a sentir cuando, por ejemplo, A tiene una actitud hacia B, o hace algo a B, que es del tipo de actitud o acción que $\rightarrow$ si $A$ la tuviese hacia mí o me la hiciera a mí- podría producir en mí resentimiento hacia A. Ahora bien, ésta no puede ser la versión adecuada, ya que puedo sentir resentimiento por muchas cosas específicas a una relación determinada y particulares a mi persona, que me ofenden a mí pero que no tendrían por qué ofender a otros y que, por tanto, no pueden generalizarse a otras personas.

¿De qué tipo deberán ser entonces las manifestaciones de la exigencia general de buena voluntad en las que se apoyan las actitudes impersonales? Las manifestaciones serán múltiples pero deberán tener una forma generalizada. Así, Bennett sugiere que "una actitud es moral sólo si se apoya en un principio general o al menos en algo que no se refiera a un ítem particular como, por ejemplo, a algo que sólo me atañe a $\mathrm{min}^{m} .{ }^{15}$

Pero si lo que distingue a las emociones reactivas per-

14 Op. cil., p. 15.

18 Bennett, op. cil., p. 46. 
sonales de las emociones reactivas morales es que las segundas se basan en principios o en generalizaciones, esto no parece suficiente para caracterizarlas como 'morales'. Hay grupos sociales que tienen principios, reglas, hábitos, códigos de conducta, etc., que no pueden caracterizarse como morales. Sin embargo, parece que la actuación de una persona perteneciente a una comunidad determinada en contra del código de conducta que rige en el grupo, puede producir indignación en los demás miembros del grupo: como cuando alguien se viste de rojo en un entierro, o actúa en contra de una regla de etiqueta. Éste es un tipo de indignación que esta gente por lo general no sentiría frente a alguien que no pertenece a esa comunidad particular. Las emociones reactivas requieren del punto de vista participativo e involucrado. Pero si para poder indignarnos basta con que alguien vaya en contra de las reglas de conducta que regulan las relaciones entre los miembros de un grupo determinado, entonces la indignación no puede ser una emoción exclusivamente moral. Puede ser impersonal, en el sentido de que no requiere de relaciones personales específicas y de que no la siente una persona esencialmente por algo que le atañe sólo a ella -aunque sí la puede sentir por algo que le atañe a ella en la medida en que es miembro de un grupo.

Ahora bien, si lo que distingue a la indignación no moral de la indignación moral es el tipo de principios o generalizaciones en las que se basa, entonces todavía falta una caracterización de los principios morales.

La referencia a la buena voluntad tampoco nos permite delimitar los casos morales. Es una referencia a algo demasiado general y su utilidad dependerá de cuáles sean las manifestaciones que se tomen en cuenta. Podríamos decir, por ejemplo, que una regla de etiqueta es una manifestación de buena voluntad en el trato que 
tienen unos con otros los miembros de una comunidad; así, podemos ver la aceptación de la regla como una manera de mostrar respeto mutuo.

Otra posibilidad es delimitar lo moral como aquello que se basa en principios o generalizaciones tan amplias que valen para todos los seres humanos, como, por ejemplo, el respeto a la vida, el evitar la crueldad y el sufrimiento inútil..., esto es, principios en los cuales, como dice Strawson, "se reafirma el sentido de simpatía y una humanidad común" ${ }^{16}$ La indignación moral sería entonces aquella emoción que sentimos cuando se violan estas exigencias generales en casos específicos. El problema con esta versión es que la ruta que va de la exigencia general a un caso particular no es directa, ya que intervienen muchos factores que dependen, entre otras cosas, de las creencias de las personas involucradas, de su apreciación de la situación y de las concepciones que tenga un individuo o una comunidad respecto de cómo han de manifestarse estas exigencias. Pero mientras no se especifique de una manera clara cómo se relacionan estas generalizaciones con sus manifestaciones específicas, de tal manera que sean aplicables a comunidades y casos, no tendremos idea de cuáles son las condiciones que hacen de las emociones impersonales casos de emociones morales.

16 Strawson, "Reply to Bennett”, p. 266. 